\title{
Influence of the combined disposal of sewage and brine on meiofauna
}

\section{Influencia de la combinación de vertidos de aguas residuales y salmuera sobre la meiofauna}

\author{
Rodrigo Riera' ${ }^{1 *}$, Fernando Tuya ${ }^{2}$, Alicia Sacramento ${ }^{1}$, Eva Ramos $^{1}$, Óscar Monterroso ${ }^{1}$, \\ Myriam Rodríguez ${ }^{1}$ \\ ${ }^{1}$ Centro de Investigaciones Medioambientales del Atlántico (CIMA SL), Arzobispo Elías Yanes, 44, \\ 38206 La Laguna, Tenerife, Canary Islands, Spain. \\ ${ }^{a}$ Current address: Dept. of Biodiversity, Qatar Environment and Energy Research Institute, 5825 Doha, Qatar. \\ 2 BIOGES, Universidad de Las Palmas de Gran Canaria, 35017 Las Palmas, Canary Islands, Spain.
}

* Corresponding author. Email: rodrigo@cimacanarias.com

\begin{abstract}
Confluence of anthropogenic influences is common in coastal areas (e.g., disposal of different pollutants like industrial and domestic sewage, brine, etc.). In this study we assessed whether the combined disposal of domestic sewage and brine altered patterns in the abundance and assemblage structure of subtidal meiofauna inhabiting sandy seabeds. Samples were collected in May 2008 and January 2009 at varying distances $(0,15$, and $30 \mathrm{~m})$ from the discharge point. Meiofaunal abundances were consistently larger at $0 \mathrm{~m}(1663.05 \pm 1076.86$ ind $10 \mathrm{~cm}^{-2}$, mean \pm standard error) than at $15 \mathrm{~m}\left(471.21 \pm 307.97\right.$ ind $\left.10 \mathrm{~cm}^{-2}\right)$ and $30 \mathrm{~m}\left(316.50 \pm 256.85\right.$ ind $\left.10 \mathrm{~cm}^{-2}\right)$ from the discharge outfall. This pattern was particularly accentuated for nematodes. Proximity to the discharge point also altered patterns in meiofaunal assemblage structure, though temporal shifts in the sedimentary composition also contributed to explain differences in the meiofaunal assemblage structure. As a result, meiofauna may be a reliable tool for monitoring studies of the combined disposal of sewage and brine as long as potential confounding factors (here temporal changes in grain size composition) are considered.
\end{abstract}

Key words: sewage, brine, meiofauna, sandy seabeds, Canary Islands.

RESUMEN. La confluencia de diferentes impactos antropogénicos es común en áreas costeras marinas; por ejemplo, el vertido conjunto de varios contaminantes (aguas residuales industriales y domésticas, salmuera, etc.) en la misma zona. En este estudio, se examinó si el vertido combinado de aguas residuales domésticas y salmuera altera los patrones de abundancia y la estructura de la comunidad meiofaunal del medio submareal. Las muestras fueron recolectadas en mayo de 2008 y enero de 2009 a 0,15 y 30 m de distancia del punto de vertido. Las abundancias meiofaunales fueron más elevadas en el punto de vertido (1663.05 \pm 1076.86 individuos $10 \mathrm{~cm}^{-2}$, media \pm error estándar) que a $15 \mathrm{~m}\left(471.21 \pm 307.97\right.$ individuos $\left.10 \mathrm{~cm}^{-2}\right)$ y $30 \mathrm{~m}$ de distancia $\left(316.50 \pm 256.85\right.$ individuos $\left.10 \mathrm{~cm}^{-2}\right)$ del punto de vertido. Este patrón de abundancia ha sido acusado particularmente para los nematodos. La proximidad al punto de vertido alteró la estructura de la comunidad meiofaunal, aunque los cambios estacionales en la composición del sedimento también contribuyeron a explicar las diferencias en las abundancias meiofaunales y en la estructura de la comunidad. Como resultado, la meiofauna puede considerarse una herramienta fiable para estudios de seguimiento de vertidos combinados de aguas residuales y salmuera, siempre y cuando la presencia de factores enmascarantes, como los cambios estacionales en la composición granulométrica del sedimento, se incluyan en los diseños.

Palabras clave: aguas residuales, salmuera, meiofauna, fondos arenosos, islas Canarias.

\section{INTRODUCTION}

In coastal environments, sewage discharge is a common source of human disturbance. Detailed studies have been conducted on the effects of sewage on several marine assemblages, such as fishes (Azzurro et al. 2010) and sessile organisms on rocky substrates (Terlizzi et al. 2002), and diverse ecosystems, including coral reefs (Reopanichkul et al. 2009), seagrass meadows (Balestri et al. 2004), and sandy unvegetated substrates (Smith and Shackley 2006). These studies have demonstrated that sewage discharges can alter patterns in the structure and diversity of recipient assemblages, though effects can notably vary depending on the response variables and data analysis (Pearson and Rosenberg

\section{INTRODUCCIÓN}

En ambientes costeros, los vertidos residuales son una fuente común de perturbación antropogénica. Se han estudiado de forma detallada los impactos de aguas residuales sobre varias comunidades marinas, como las de peces (Azzurro et al. 2010) y organismos sésiles sobre sustratos rocosos (Terlizzi et al. 2002), y sobre diferentes ecosistemas, como arrecifes de coral (Reopanichkul et al. 2009), praderas de fanerógamas marinas (Balestri et al. 2004) y sustratos arenosos sin vegetación (Smith y Shackley 2006). Estos trabajos han demostrado que los vertidos residuales pueden alterar patrones en la estructura de aguas y la diversidad de las comunidades afectadas, aunque los efectos pueden variar 
1978, Guidetti et al. 2003, Chapman et al. 2005). Sewage discharges alter the organic content and biochemical composition of sediments (Cotano and Villate 2006), as well as the biochemical properties of the water column (Oviatt et al. 1987), affecting biological assemblages through blooms of nitrogen-consuming algae (Thornberg et al. 2008); high concentrations of pathogens and bacteria that may enter the food chain (Korajkic et al. 2010); reef erosion by mechanical, chemical, and biological processes (Pastorok and Bilyard 1985); changes in protein synthesis and growth (Houlihan et al. 1994); changes in feeding and respiration rates (Butler et al. 1990); and inhibition of germination (Burridge et al. 1996).

In the last decades, the number of desalination plants has increased in arid and semi-arid areas. Desalination plants extract large volumes of seawater and discharge hypersaline brine back into the marine environment (Roberts et al. 2010). Former studies have identified several potential mechanisms by which brine may impact marine ecosystems and their organisms (Sadhwani et al. 2005, Gacia et al. 2007, Riera et al. 2011b).

Meiofaunal organisms are a group of animals appropriately suited for environmental impact assessment because of the peculiarities of their life cycles: small size, high turnover, and lack of larval dispersion (Higgins and Thiel 1988). For example, meiofauna are sensitive to environmental disturbances caused by a range of impacts, from organic enrichment to mining (Austen et al. 1994, Danovaro et al. 1995, Mirto et al. 2000, Grego et al. 2009). Several studies have analyzed responses of meiofaunal assemblages to sewage discharges (Vidakovic 1983; Sandulli and De Nicola-Giudici 1990, 1991; Fraschetti et al. 2006). These studies have shown significant changes in meiofaunal assemblage structure in the vicinity of sewage discharges, including an increase in the abundance of nematodes and a concurrent decrease in the abundance of harpacticoid copepods (Sandulli and De Nicola-Giudici 1990, 1991). An overall decrease in the richness of meiofaunal taxa has also been detected (Fraschetti et al. 2006). Meiofaunal assemblages can also be affected by the release of brine. For example, proximity to a brine discharge point affected the abundance of meiofauna: the lowest abundances of meiofauna, particularly nematodes and copepods, were observed immediately adjacent to a brine discharge point than 15 and $30 \mathrm{~m}$ away (Riera et al. 2011b).

A convergence of anthropogenic impacts is common in coastal areas (e.g., disposal of different pollutants, including industrial and domestic sewage, brine, etc.); however, studies that have tried to account for changes in the assemblage structure and abundance of recipient assemblages as a result of the combined disposal of pollutants are lacking, particularly for meiofauna. In this study, we took advantage of the release of domestic sewage and brine through the same outfall to assess whether meiofaunal abundance and assemblage structure changed with varying distance from the discharge point. notablemente dependiendo de las variables de respuesta y el análisis de los datos (Pearson y Rosenberg 1978, Guidetti et al. 2003, Chapman et al. 2005). Las aguas residuales alteran el contenido orgánico y la composición bioquímica de los sedimentos (Cotano y Villate 2006), así como las propiedades bioquímicas de la columna de agua (Oviatt et al. 1987), afectando las comunidades biológicas mediante explosiones de algas consumidoras de nitrógeno (Thornberg et al. 2008), concentraciones elevadas de patógenos y bacterias que pueden incorporarse a la cadena alimentaria (Korajkic et al. 2010), erosión del arrecife por procesos mecánicos, bioquímicos y biológicos (Pastorok y Bilyard 1985), cambios en la síntesis de proteínas y crecimiento (Houlihan et al. 1994), cambios en las tasas de respiración y alimentación (Butler et al. 1990) e inhibición de la germinación (Burridge et al. 1996).

En las últimas décadas, el número de plantas desalinizadoras se ha incrementado en áreas áridas y semiáridas del planeta. Las plantas desalinizadoras extraen grandes volúmenes de agua salada y descargan agua hipersalina (salmuera) de vuelta al medio marino (Roberts et al. 2010). Varios estudios anteriores han identificado varios mecanismos potenciales por los cuales la salmuera puede impactar los ecosistemas marinos y sus organismos (Sadhwani et al. 2005, Gacia et al. 2007, Riera et al. 2011b).

Los organismos meiofaunales son un grupo de animales adecuados para los seguimientos ambientales, por las peculiaridades de sus ciclos de vida: talla pequeña, potencial reproductor alto y ausencia de dispersión larvaria (Higgins y Thiel 1988). Por ejemplo, la meiofauna es sensible a las alteraciones ambientales causadas por diferentes tipos de impactos, desde el enriquecimiento orgánico hasta las actividades de la minería (Austen et al. 1994, Danovaro et al. 1995, Mirto et al. 2000, Grego et al. 2009). Algunos estudios han analizado las respuestas de las comunidades meiofaunales a los vertidos de aguas residuales (Vidakovic 1983; Sandulli y De Nicola-Giudici 1990, 1991; Fraschetti et al. 2006). Estos estudios han mostrado cambios significativos en la estructura de la comunidad meiofaunal en los alrededores de los vertidos residuales, incluyendo un incremento en la abundancia de nematodos y una disminución simultánea en la abundancia de copépodos harpacticoides (Sandulli y De Nicola-Giudici 1990, 1991). También se ha detectado una disminución global en la riqueza de los grupos meiofaunales (Fraschetti et al. 2006). Las comunidades meiofaunales pueden encontrarse afectadas por los vertidos de salmuera; por ejemplo, la proximidad a un punto de descarga de salmuera afectó la abundancia de la meiofauna: las densidades mínimas de meiofauna, especialmente de nematodos y copépodos, fueron observadas inmediatamente adyacentes al punto de descarga de la salmuera, en comparación con los puntos localizados a 15 y $30 \mathrm{~m}$ de distancia (Riera et al. 2011b).

La confluencia de impactos antropogénicos es común en áreas costeras (e.g., el vertido de diferentes contaminantes, 


\section{MATERIAL AND METHODS}

\section{Study area and sampling design}

This study was conducted in the vicinity of the Tarajalillo treatment plant, located on the south coast of Gran Canaria $\left(27^{\circ} 77^{\prime} 45^{\prime \prime} \mathrm{N}, 15^{\circ} 51^{\prime} 90^{\prime \prime} \mathrm{W}\right)$, Spain (fig. 1). The plant has a brine and domestic sewage outfall of approximately $500 \mathrm{~m}$ running offshore. The outfall diameter is $\sim 25 \mathrm{~cm}$ and discharges through a "mouth" on a sandy seabed at $10 \mathrm{~m}$ depth. Disposal of brine is continuous, while domestic sewage is occasionally dumped as well (ca. $10-50,000 \mathrm{~m}^{3}$ per month). Salinity at the discharge point typically ranges from 49 to 53 , whereas $30 \mathrm{~m}$ away, it ranges from 36.6 to 36.7, which are natural levels (table 1).

Samples were collected at a distance of 0,15 , and $30 \mathrm{~m}$ from the brine discharge point. Collections at $0 \mathrm{~m}$ were immediately continuous to the discharge outfall. Samples collected $30 \mathrm{~m}$ from the outfall were used as control because of the natural salinity levels recorded there. This criterion, however, did not take into account the organic content of the sediments. Data prior to the installation of the outfall are nonexistent; hence, no hypothesis concerning putative temporal variation of meiofaunal assemblages (i.e., before $v s$ after the presence of the outfall) can be made.

Sediment cores $\left(3.6 \mathrm{~cm}\right.$ inner diameter, area $10 \mathrm{~cm}^{2}$ ) were hammered into the sediment to a depth of $30 \mathrm{~cm}$. Fifteen replicates were randomly collected for faunal determinations at each distance. Three cores at each distance were additionally collected for analysis of sediment features (see below). The level of replication was based on a previous study (Riera et al. 2011a). Sampling was conducted twice, on May 2008 and January 2009, to assess whether meiofaunal patterns with varying proximity to the discharge point were temporally consistent at two randomly-selected times.

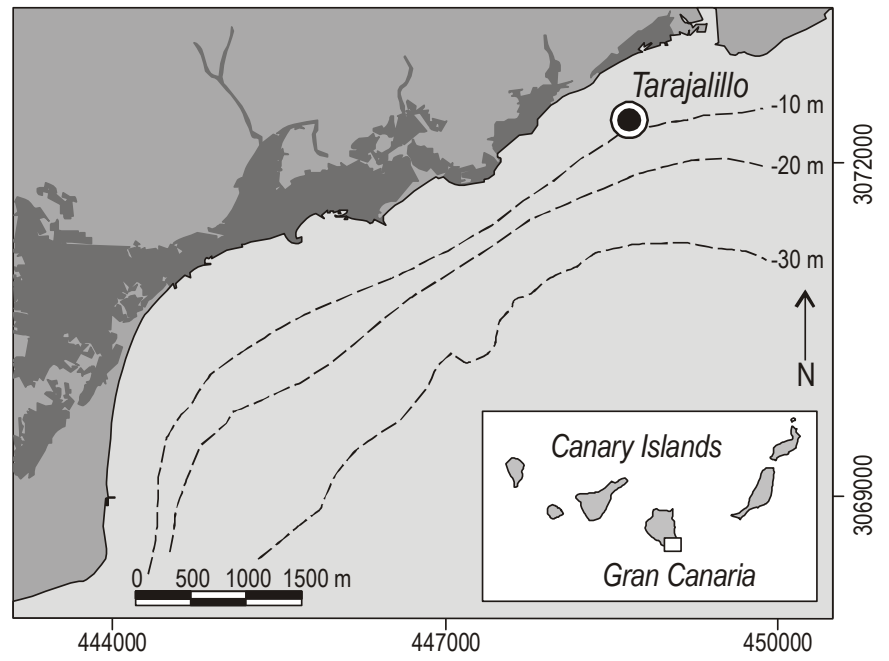

Figure 1. Map of the study area.

Figura 1. Mapa del área de estudio. incluyendo las aguas residuales industriales, domésticas, salmuera, etc.). Sin embargo, estudios sobre los cambios en la estructura y abundancia de las comunidades afectadas por la contaminación combinada de varios vertidos, particularmente sobre las comunidades meiofaunales, son muy escasos. En este estudio, se aprovechó el vertido de aguas residuales domésticas y salmuera a lo largo del mismo punto de vertido para establecer si las abundancias meiofaunales y la estructura de la comunidad cambian según su proximidad al punto de descarga.

\section{MATERIALES Y MÉTODOS}

\section{Área de estudio y diseño de muestreo}

El estudio se realizó alrededor de la planta de tratamiento Tarajalillo, localizada en la costa sur de la isla de Gran Canaria $\left(27^{\circ} 77^{\prime} 45^{\prime \prime} \mathrm{N}, 15^{\circ} 51^{\prime} 90^{\prime \prime}\right.$ O) (fig. 1). La planta vierte salmuera y aguas residuales domésticas en el punto de descarga, situado a unos $500 \mathrm{~m}$ mar adentro. El diámetro de la conducción (emisario submarino) es de $\sim 25 \mathrm{~cm}$ y descarga sobre un fondo arenoso situado a $10 \mathrm{~m}$ de profundidad. El vertido de salmuera es continuo a lo largo del tiempo y el de aguas residuales domésticas es ocasional (caudal aproximado de $10-50,000 \mathrm{~m}^{3}$ al mes). La salinidad en el punto de descarga varía entre 49 y 53 , mientras que a $30 \mathrm{~m}$ de distancia la salinidad se mantiene en los intervalos naturales, entre 36.6 y 36.7 (tabla 1).

La recolección de muestras se realizó a 0,15 y $30 \mathrm{~m}$ de distancia del punto de vertido de salmuera. Las muestras a $0 \mathrm{~m}$ fueron recolectadas en fondos inmediatamente adyacentes a la boca del emisario. Las muestras recolectadas a $30 \mathrm{~m}$ de distancia se consideraron como control, porque la salinidad varió entre sus intervalos naturales; sin embargo, este criterio no se tomó en cuenta para el contenido orgánico de los sedimentos. No existen datos previos a la instalación del punto de descarga; por tanto, no se pudieron realizar hipótesis sobre la posible variación temporal de las poblaciones meiofaunales (e.g., comparación entre antes y después de la presencia del emisario).

Se clavaron tubos (cores; $3.6 \mathrm{~cm}$ de diámetro interno, área de $10 \mathrm{~cm}^{2}$ ) con un martillo, hasta una profundidad de $30 \mathrm{~cm}$, para la recolección de sedimento. Un total de 15 réplicas fueron recolectadas de forma aleatoria para la determinación faunística en cada una de las distancias. Tres tubos fueron recogidos adicionalmente en cada una de las distancias para el análisis de las características sedimentarias (ver abajo). El nivel de replicación está basado en un estudio previo (Riera et al. 2011a). El muestreo se llevó a cabo dos veces, en mayo de 2008 y enero de 2009, para determinar si los patrones meiofaunales variaban, según su proximidad al punto de descarga, de forma consistente durante dos períodos seleccionados de forma aleatoria. 


\section{Analysis of environmental variables}

At each distance, salinity, temperature, $\mathrm{pH}$, and chlorophyll $a$ were measured, at 1-m intervals throughout the water column, using a CTD (SBE 19plusV2 sn5198); mean values were then calculated for each distance. Porewater salinity was measured by a salinity meter (WTW LF95/SET).

Since sediment features can notably influence softbottom meiofaunal assemblages (Gray 1981, Pearson and Rosenberg 1978), we quantified five sediment-related variables (sediment grain size, organic matter, total nitrogen, total phosphorus, and microbiology) to estimate their potential effects on the patterns of abundance and assemblage structure of the community in relation to the proximity to the discharge point. After thawing, sediment samples were sieved through a $0.5-\mathrm{mm}$ sieve, and the $<0.5 \mathrm{~mm}$ fraction was oven dried at $90{ }^{\circ} \mathrm{C}$ for $24 \mathrm{~h}$. This fraction was then drysieved at $0.5 \varphi$ intervals, down to $1.0 \varphi(0.5 \mathrm{~mm})$. The $<0.5 \mathrm{~mm}$ fraction was freeze-dried and analyzed on a Coulter LS130 laser sizer. The laser sizer results were combined with the dry sieve results to give the full particle size distribution. The method described by Walkley and Black (1934) was used to calculate the organic matter content of the sediment by acid rapid dichromate oxidation. Total nitrogen in sediments was determined following the Kjeldahl method (Bradstreet 1965) and total phosphorus in sediments was calculated using a spectrophotometric method (Murphy and Riley 1962). Escherichia coli was measured by calculating total coliforms using the standard $\mathrm{mFC}$ medium for faecal coliform detection with transfer to NA + MUG plates for incubation at $35^{\circ} \mathrm{C}$ for $4 \mathrm{~h}$. Total coliforms were measured using the mEndo LEs medium for incubation at $35^{\circ} \mathrm{C}$.

\section{Analysis of meiofauna}

Samples were preserved in a $10 \%$ seawater-formaldehyde solution and subsequently decanted through a $0.063-\mathrm{mm}$ sieve. The remaining fraction was separated into different taxonomic groups under a binocular microscope, and preserved in $70 \%$ ethanol. Meiofaunal specimens were determined to major taxon level using a binocular microscope or a LEICA DMLB microscope equipped with Nomarski interference contrast (Somerfield and Warwick 1996).

\section{Statistical analysis}

Differences in the meiofaunal assemblage structure with varying distance from the discharge point (i.e., 0, 15, or $30 \mathrm{~m}$ ) and the surveys (May 2008 vs January 2009) were tested by a permutational multivariate analysis of variance (PERMANOVA) that included two factors: Distance (fixed factor) and Year (random factor, orthogonal to Distance). The same model, but in a univariate context, tested for differences in overall meiofaunal abundance and the abundance of the dominant meiofaunal groups (nematodes and copepods,

\section{Análisis de las variables ambientales}

Para cada distancia, se determinó la salinidad, la temperatura, el $\mathrm{pH}$ y la clorofila $a$ con un CTD (SBE 19plusV2 sn5198) a intervalos de $1 \mathrm{~m}$ a lo largo de la columna de agua; posteriormente, se calcularon los valores medios para cada distancia. La salinidad intersticial se midió con un salinómetro (WTW LF 95/SET).

Debido a que las características del sedimento pueden influir de forma notable sobre las comunidades meiofaunales de fondos blandos (Gray 1981, Pearson y Rosenberg 1978), se cuantificaron cinco variables relacionadas con el sedimento (granulometría, materia orgánica, nitrógeno total, fósforo total y microbiología del sedimento) para estimar los potenciales efectos sobre los patrones de abundancia y estructura de la comunidad con la proximidad al punto de vertido. Después de la desecación, las muestras de sedimento fueron tamizadas con un tamiz con luz de malla de $0.5 \mathrm{~mm}$ de diámetro, y la fracción inferior a este diámetro fue secada en un horno a $90{ }^{\circ} \mathrm{C}$ durante $24 \mathrm{~h}$. Esta fracción fue tamizada a intervalos de $0.5 \varphi$, hasta $1.0 \varphi(0.5 \mathrm{~mm})$. La fracción inferior a $0.5 \mathrm{~mm}$ fue desecada y homogeneizada para ser analizada por un medidor láser Coulter LS130. Los resultados del medidor láser fueron combinados con los resultados del tamizado para determinar la distribución del diámetro de la partícula de sedimento. El método de Walkley y Black (1934) fue utilizado para calcular el contenido en materia orgánica del sedimento, a través de una oxidación ácida rápida con dicromato. El nitrógeno total sedimentario fue determinando según el método Kjeldahl (Bradstreet 1965) y el contenido total de fósforo sedimentario, mediante un método espectrofotométrico (Murphy y Riley 1962). La presencia de Escherichia coli fue determinada a partir del cálculo de coliformes totales utilizando el medio estándar mFC para coliformes fecales, con trasferencia a placas NA + MUG para después incubarlas a $35^{\circ} \mathrm{C}$ durante $24 \mathrm{~h}$. Los coliformes totales fueron determinados a partir del medio m-Endo LES por incubación a $35^{\circ} \mathrm{C}$.

\section{Análisis de la meiofauna}

Las muestras fueron conservadas en una solución de formaldehido al $10 \%$ y posteriormente fueron tamizadas (luz de malla de $0.063 \mathrm{~mm}$ ). La fracción remanente fue separada en diferentes grupos taxonómicos bajo una lupa y preservada en etanol al $70 \%$. Los ejemplares meiofaunales fueron determinados a nivel de grupo taxonómico con un estereomicroscopio e incluso un microscopio óptico Leica DMLB equipado con contraste interferencial de Nomarski (Somerfield y Warwick 1996).

\section{Análisis estadístico}

Las diferencias en la estructura de la comunidad meiofaunal según su proximidad al punto de vertido (distancias: 0, 15 
Table 1. Mean values ( \pm standard error) of environmental variables with varying proximity $(0,15$, and $30 \mathrm{~m})$ to the discharge point. Tabla 1. Valores medios ( \pm error estándar) de las variables ambientales según su proximdad $(0,15$ y $30 \mathrm{~m})$ al punto de descarga.

\begin{tabular}{|c|c|c|c|}
\hline & $0 \mathrm{~m}$ & $15 \mathrm{~m}$ & $30 \mathrm{~m}$ \\
\hline Pore water salinity & $48.70 \pm 1.40$ & $38.90 \pm 0.90$ & $36.70 \pm 0.20$ \\
\hline Water column salinity & $50.80 \pm 3.50$ & $39.00 \pm 0.80$ & $36.60 \pm 0.10$ \\
\hline Water column temperature $\left({ }^{\circ} \mathrm{C}\right)$ & $20.93 \pm 0.50$ & $20.80 \pm 0.40$ & $20.60 \pm 0.30$ \\
\hline Chlorophyll $\left(\mu \mathrm{g} \mathrm{L}^{-1}\right)$ & $1.40 \pm 0.40$ & $1.10 \pm 0.20$ & $0.90 \pm 0.30$ \\
\hline Sediment: total nitrogen $\left(\mathrm{mg} \mathrm{kg}^{-1}\right)$ & $<1.00$ & $<1.00$ & $<1.00$ \\
\hline Sediment: total phosphorus $\left(\mathrm{mg} \mathrm{kg}^{-1}\right)$ & $2.66 \pm 1.04$ & $2.87 \pm 0.47$ & $1.30 \pm 0.52$ \\
\hline Sediment: organic matter $(\%)$ & $0.64 \pm 0.18$ & $0.25 \pm 0.07$ & $0.32 \pm 0.06$ \\
\hline Sediment: gravels $(\%)$ & $18.91 \pm 18.07$ & $0.44 \pm 0.39$ & $0.23 \pm 0.40$ \\
\hline Sediment: very coarse sands $(\%)$ & $19.67 \pm 7.47$ & $0.35 \pm 0.31$ & $0.30 \pm 0.36$ \\
\hline Sediment: coarse sands $(\%)$ & $14.69 \pm 8.74$ & $0.48 \pm 0.45$ & $0.23 \pm 0.40$ \\
\hline Sediment: medium sands (\%) & $2.99 \pm 1.42$ & $0.88 \pm 0.95$ & $0.53 \pm 0.32$ \\
\hline Sediment: fine sands (\%) & $11.66 \pm 5.38$ & $25.49 \pm 12.97$ & $25.50 \pm 13.06$ \\
\hline Sediment: very fine sands $(\%)$ & $23.00 \pm 9.42$ & $57.32 \pm 8.10$ & $57.28 \pm 4.67$ \\
\hline Sediment: silt/clay (\%) & $9.06 \pm 4.90$ & $15.04 \pm 4.73$ & $15.92 \pm 8.19$ \\
\hline \multicolumn{4}{|l|}{ January 2009} \\
\hline Sediment: total nitrogen $\left(\mathrm{mg} \mathrm{kg}^{-1}\right)$ & $1.30 \pm 0.10$ & $1.23 \pm 0.06$ & $1.25 \pm 0.07$ \\
\hline Sediment: total phosphorus $\left(\mathrm{mg} \mathrm{kg}^{-1}\right)$ & $3.60 \pm 0.35$ & $4.03 \pm 1.88$ & $3.13 \pm 0.85$ \\
\hline Sediment: organic matter $(\%)$ & $0.63 \pm 0.38$ & $0.86 \pm 0.56$ & $1.26 \pm 0.53$ \\
\hline Total coliforms (CFU $100 \mathrm{~mL}^{-1}$ ) & $130.00 \pm 35.37$ & $116.00 \pm 26.46$ & $181.67 \pm 27.30$ \\
\hline Escherichia coli (CFU $\left.100 \mathrm{~mL}^{-1}\right)$ & $0.67 \pm 1.15$ & $23.33 \pm 40.41$ & $1.00 \pm 1.73$ \\
\hline Sediment: gravels $(\%)$ & $6.39 \pm 2.95$ & $9.93 \pm 5.17$ & $19.26 \pm 7.16$ \\
\hline Sediment: very coarse sands (\%) & $12.71 \pm 4.22$ & $11.66 \pm 5.53$ & $26.11 \pm 1.63$ \\
\hline Sediment: coarse sands $(\%)$ & $9.84 \pm 1.80$ & $8.20 \pm 3.73$ & $14.82 \pm 3.53$ \\
\hline Sediment: medium sands (\%) & $4.26 \pm 0.32$ & $6.02 \pm 1.41$ & $5.08 \pm 0.42$ \\
\hline Sediment: fine sands (\%) & $27.83 \pm 1.70$ & $31.85 \pm 7.64$ & $16.43 \pm 3.85$ \\
\hline Sediment: very fine sands $(\%)$ & $38.61 \pm 6.67$ & $31.80 \pm 2.49$ & $17.84 \pm 2.98$ \\
\hline Sediment: silt/clay (\%) & $0.37 \pm 0.20$ & $0.55 \pm 0.16$ & $0.46 \pm 0.05$ \\
\hline
\end{tabular}


which accounted for $97.4 \%$ of the organisms). Though variances remained heterogeneous in all cases, an increase in a type I error was decreased by reducing the $\alpha$ value to the 0.01 level (Underwood 1991); ANOVA is robust to such departures for balanced studies. Post-hoc tests were used, when appropriate, to resolve differences among distances from the discharge point.

A distance-based redundancy analysis (db-RDA, Legendre and Anderson 1999) tested whether the variation in environmental variables significantly contributed to explain the variation in the meiofaunal assemblage structure with varying proximity to the discharge point. Multivariate multiple regression, using the DISTLM routine (Anderson 2001), tested the significance of these relationships by fitting a linear model based on Bray-Curtis dissimilarities on square-root transformed abundance data. To retain variables with good explanatory power as a result of strong colinearity among variables (determined through a matrix of Pearson product-moment correlation coefficients), the Akaike information criterion (AIC) was used as a selection criterion (the smaller the value the better the model, Legendre and Anderson 1999). Analyses were based on a "forward" selection procedure. All multivariate procedures were carried out using the PRIMER 6.0 and PERMANOVA+ statistical package.

\section{RESULTS}

A total of 44,114 individuals were collected, belonging to 14 taxonomic groups: Amphipoda, Bivalvia, Copepoda, Cumacea, Decapoda, Isopoda, Misidacea, Nematoda, Oligochaeta, Ostracoda, Polychaeta, Sipuncula, Tanaidacea, and Turbellaria. The most abundant group was nematodes, which overwhelmingly dominated the meiofaunal assemblage (41,335 individuals, $93.7 \%$ of the overall abundance), followed by copepods (1,650 individuals, $3.7 \%$ ). Decapods and sipunculids, on the other hand, were scarce, with one single individual.

Overall meiofaunal abundance was affected by proximity to the discharge outfall: abundances at $0 \mathrm{~m}(1663.05 \pm$ 1076.86 ind $10 \mathrm{~cm}^{-2}$ ) were larger than at 15 and $30 \mathrm{~m}$ from the discharge point $\left(15 \mathrm{~m}: 471.21 \pm 307.97\right.$ ind $10 \mathrm{~cm}^{-2}$; $30 \mathrm{~m}: 316.50 \pm 256.85$ ind $10 \mathrm{~cm}^{-2}$ ) (table 2, fig. 2a; Distance, $P=0.0096$, table 3 ) consistently between surveys (Distance $\times$ Year, $P=0.658$, table 3). Overall meiofaunal abundance was, however, significantly larger in January 2009 $\left(1014.96 \pm 683.71\right.$ ind $\left.10 \mathrm{~cm}^{-2}\right)$ than in May $2008(618.89 \pm$ 426.42 ind $10 \mathrm{~cm}^{-2}$ ) (table 2, fig. 2a; Year, $P=0.0104$, table 3). Nematodes also had larger abundances at $0 \mathrm{~m}$ $\left(1585.67 \pm 1054.57\right.$ ind $\left.10 \mathrm{~cm}^{-2}\right)$ than at $15 \mathrm{~m}(386.94 \pm$ 219.36 ind $\left.10 \mathrm{~cm}^{-2}\right)$ and $30 \mathrm{~m}\left(278.03 \pm 249.46\right.$ ind $\left.10 \mathrm{~cm}^{-2}\right)$ from the discharge point irrespective of years (fig. 2b; Distance, $P=0.0110$, Distance $\times$ Year, $P=0.678$, table 3). Copepods, however, showed a different pattern: the magnitude of differences in copepod abundance with varying proximity to the outfall was inconsistent between surveys y $30 \mathrm{~m}$ ) y campañas (mayo de $2008 v s$. enero de 2009) fueron analizadas a través de un análisis multivariante de la varianza por permutaciones (PERMANOVA) que incluyó los siguientes factores: Distancia (factor fijo) y Año (factor aleatorio, ortogonal a Distancia). El mismo modelo, pero en un contexto univariante, se empleó para las diferencias en la abundancia total meiofaunal y la abundancia de los grupos meiofaunales dominantes (nematodos y copépodos, que conformaron el $97.4 \%$ de los individuos). A pesar de que las varianzas fueron heterogéneas en todos los casos, se disminuyó la incidencia del error tipo I con la reducción del valor de $\alpha$ a 0.01 (Underwood 1991); ANOVA es una técnica robusta para estudios balanceados. Las pruebas post-hoc fueron utilizadas, en aquellos casos apropiados, para dilucidar las diferencias entre distancias al punto de descarga.

El análisis de redundancia basado en distancias (db-RDA, Legendre y Anderson 1999) se empleó para determinar si la variación en las variables ambientales contribuían de forma significativa a explicar la variabilidad en la estructura de la comunidad meiofaunal según su proximidad al punto de descarga. Una regresión múltiple multivariante, utilizando la rutina DISTLM (Anderson 2001), contrastó la significación de estas relaciones mediante un modelo lineal basado en disimilitudes de Bray-Curtis, en el cual se transformaron los datos de abundancias a la raíz cuadrada. Para retener las variables con mayor capacidad de predicción, como resultado de la fuerte colinealidad entre algunas variables, se empleó la rutina del criterio de información de Akaike (AIC) como un criterio de selección (cuanto más bajo el valor, mejor el modelo; Legendre y Anderson 1999). Los análisis estuvieron basados en un procedimiento de selección secuencial. Todos los análisis multivariantes fueron realizados con el programa PRIMER 6.0 y el paquete estadístico PERMANOVA+.

\section{RESUltados}

Se recolectaron un total de 44,114 individuos, pertenecientes a 14 grupos taxonómicos, que fueron los siguientes: Amphipoda, Bivalvia, Copepoda, Cumacea, Decapoda, Isopoda, Misidacea, Nematoda, Oligochaeta, Ostracoda, Polychaeta, Sipuncula, Tanaidacea y Turbellaria. El grupo más abundante fue el de los nematodos, que dominaron de forma clara la comunidad meiofaunal $(41,335$ individuos, $93.7 \%$ de la abundancia total), seguidos por los copépodos (1650 individuos, 3.7\%). En cambio, los decápodos y sipuncúlidos fueron minoritarios, representados por un único ejemplar.

La abundancia total meiofaunal varió según su proximidad al punto de vertido; la abundancia a $0 \mathrm{~m}(1663.05 \pm$ 1076.86 individuos $10 \mathrm{~cm}^{-2}$ ) fue más elevada que a 15 $\left(471.21 \pm 307.97\right.$ individuos $\left.10 \mathrm{~cm}^{-2}\right)$ y $30 \mathrm{~m}(316.50 \pm$ 256.85 individuos $\left.10 \mathrm{~cm}^{-2}\right)$ de distancia del punto de descarga (tabla 2, fig. 2a) (Distancia, $P=0.0096$; tabla 3), y este aumento fue consistente entre las dos campañas de muestreo (Distancia $\times$ Año, $P=0.6584$; tabla 3 ). La abundancia 
Table 2. Mean abundances ( \pm standard error) of meiofaunal groups $\left(10 \mathrm{~cm}^{-2}\right)$ with varying proximity $(0,15$, and $30 \mathrm{~m})$ to the discharge point. Tabla 2. Abundancias medias ( \pm error estándar) de grupos meiofaunales $\left(10 \mathrm{~cm}^{-2}\right)$ según su proximidad $(0,15$ y $30 \mathrm{~m})$ al punto de descarga

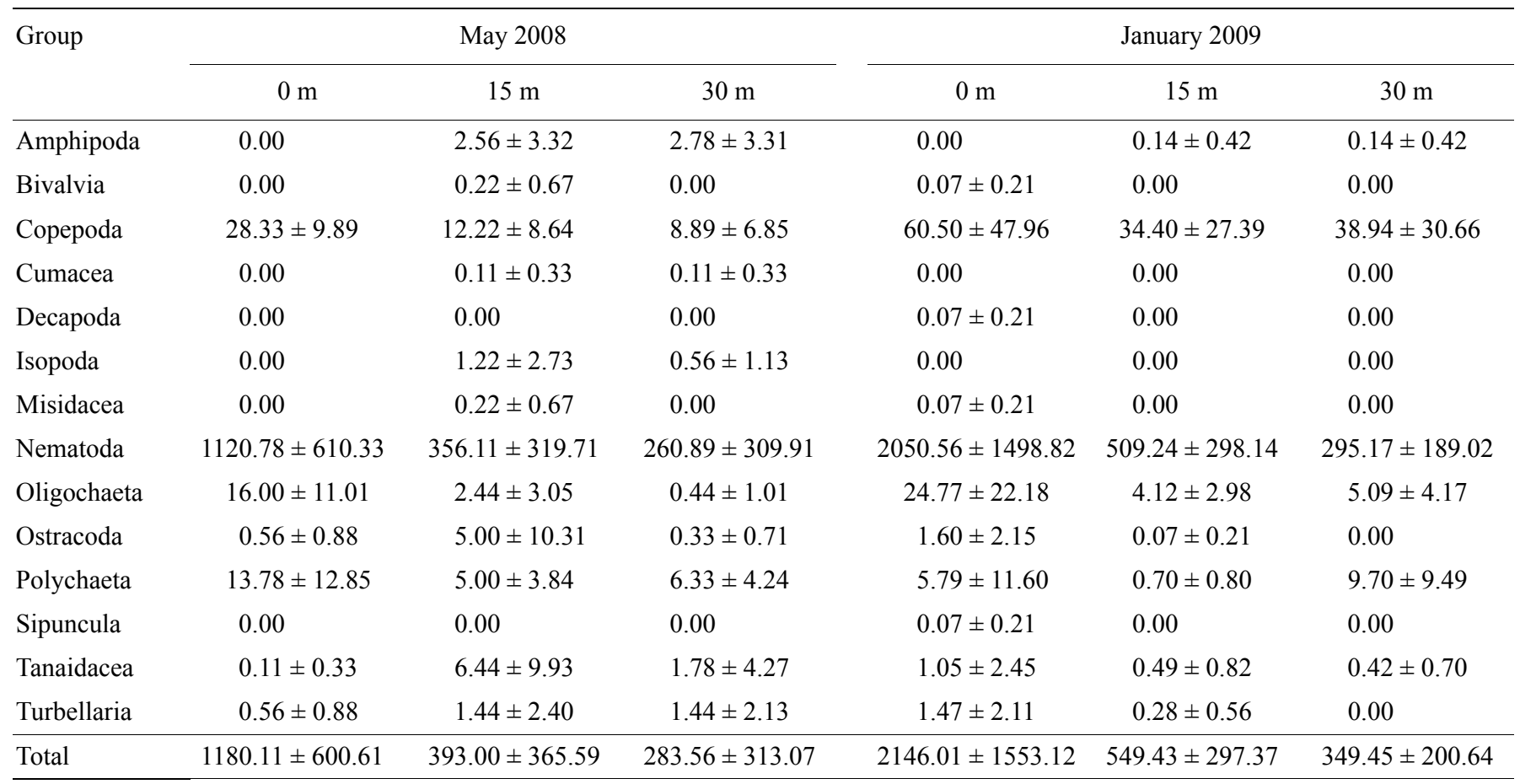

Table 3. Results of multi- and univariate ANOVA testing for differences in meiofaunal assemblage structure, overall meiofaunal abundance, and nematode and copepod abundances with varying proximity to the discharge point (Distance, fixed factor) through successive years (Year, random factor, orthogonal to Distance). $P$ values in bold denote significant values $(P<0.01)$. Data were square-root transformed.

Tabla 3. Resultados de ANOVA multi- y univariante sobre las diferencias en la estructura de la comunidad meiofaunal, la abundancia meiofaunal total, y las abundancias de nematodos y copépodos según su proximidad al punto de descarga (Distancia, factor fijo) durante años sucesivos (Año, factor aleatorio, ortogonal a Distancia). Valores de $P$ en negritas indican significación $(P<0.01)$. El asterisco indica datos transformados a la raíz cuadrada.

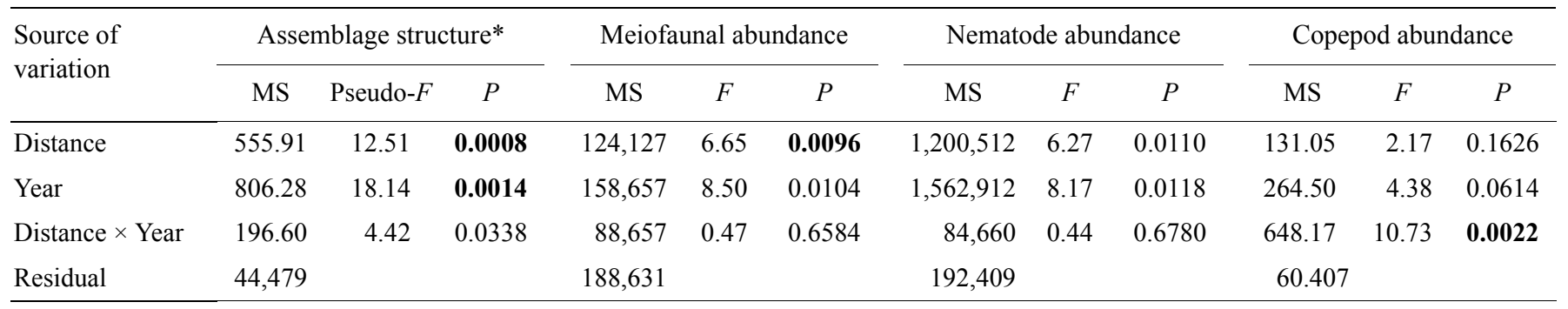

(Distance $\times$ Year, $P=0.0022$, table 3) and masked differences among the sampling distances (fig. 2c; Distance, $P=$ 0.1626 , table 3). Copepod abundance in January 2009 was larger than in May 2008, though this change was slightly non-significant (fig. 2c; Year, $P=0.0614$, table 3).

Distance from the disposal point also significantly influenced patterns of meiofaunal assemblage structure (PERMANOVA, Distance, $P=0.0008$, table 3 ), though the magnitude of these differences was not consistent between years (Distance $\times$ Year, $P=0.0338$, table 3 ). The first two axes from the db-RDA explained about $45 \%$ of the total variation in meiofaunal assemblage structure (fig. 3). The first axis was positively correlated to the percentage of silt/clay meiofaunal total fue, sin embargo, más alta en enero de 2009 $\left(1014.96 \pm 683.71\right.$ individuos $\left.10 \mathrm{~cm}^{-2}\right)$ que en mayo de 2008 $\left(618.89 \pm 426.42\right.$ individuos $\left.10 \mathrm{~cm}^{-2}\right)$ (tabla 2, figura 2a) (Año, $P=0.0104$; tabla 3 ). Los nematodos mostraron abundancias más altas a $0 \mathrm{~m}(1585.67 \pm 1054.57$ individuos $\left.10 \mathrm{~cm}^{-2}\right)$ que a $15\left(386.94 \pm 219.36\right.$ individuos $\left.10 \mathrm{~cm}^{-2}\right) \mathrm{y}$ $30 \mathrm{~m}\left(278.03 \pm 249.46\right.$ individuos $\left.10 \mathrm{~cm}^{-2}\right)$ de distancia del punto de descarga, pero no fueron consistentes entre años (fig. 2b) (Distancia, $P=0.0110$; Distancia $\times$ Año, $P=$ 0.6780; tabla 3). Por el contrario, los copépodos mostraron un patrón diferente, con diferencias inconsistentes en la abundancia entre años según la proximidad al punto de vertido (Distancia $\times$ Año, $P=0.0022$; tabla 3 ), enmascarando 

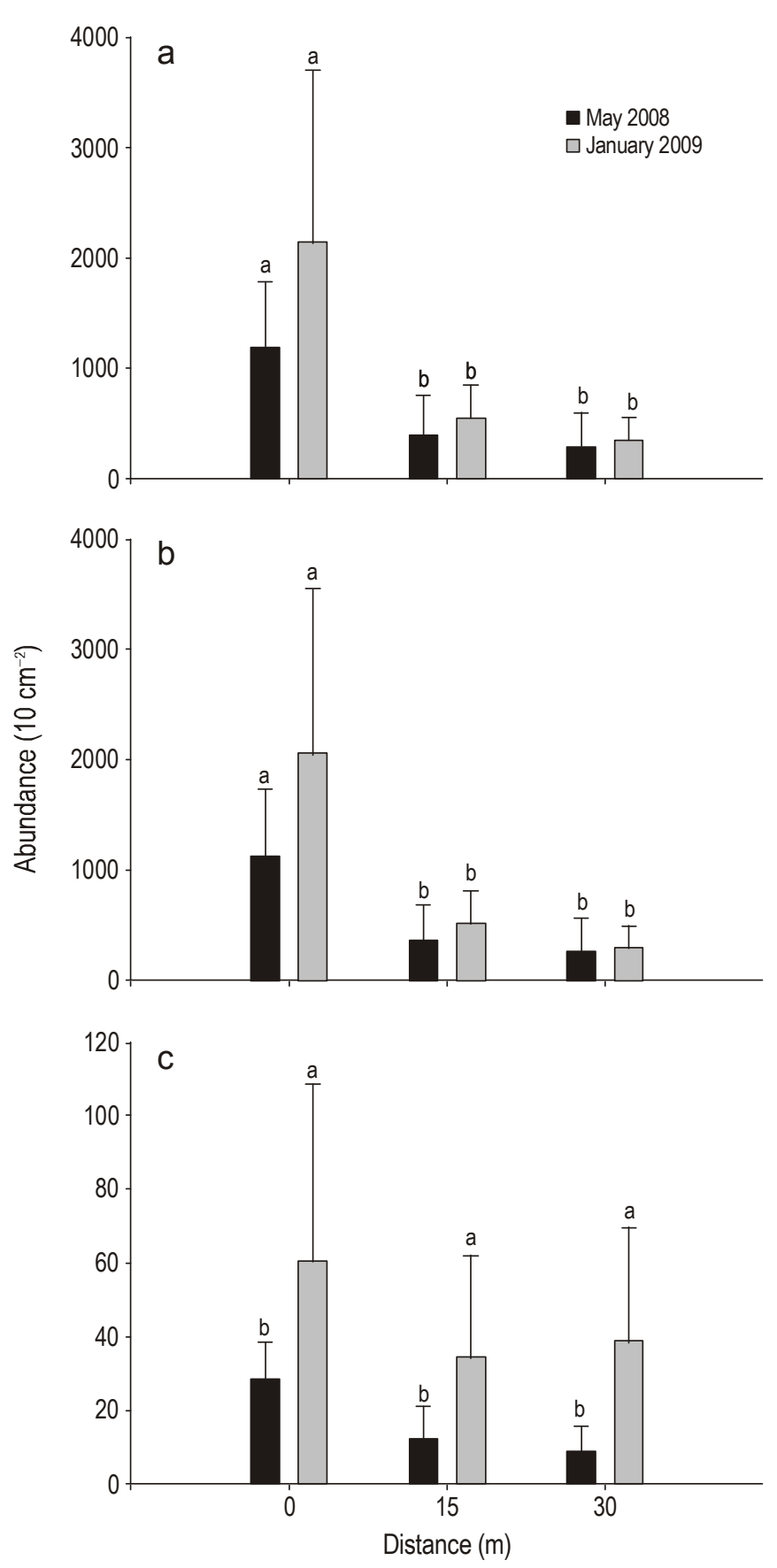

Figure 2. Meiofaunal abundance ( \pm standard error) at a distance of 0,15 , and $30 \mathrm{~m}$ from the discharge point: (a) overall meiofaunal abundance, (b) total nematode abundance, and (c) total copepod abundance. Different letters above bars denote significant differences $(P<0.05)$ among distances separately for each year.

Figura 2. Abundancias meiofauanles ( \pm error estándar) a 0,15 y $30 \mathrm{~m}$ de distancia del punto de descarga; (a) abundancia meiofaunal total, (b) abundancia total de nematodos y (c) abundancia total de copépodos. Las diferentes letras indican diferencias significativas $(P<0.05)$ entre distancias independientemente de cada año. las diferencias entre los diferentes puntos de muestreo $(0,15$ y $30 \mathrm{~m}$ ) (fig. 2c; Distancia, $P=0.1626$; tabla 3 ). La abundancia de copépodos en enero de 2009 fue más elevada que en mayo de 2008, aunque estas diferencias se encontraron en el límite de la significación (fig. 2c; Año, $P=0.0614$; tabla 3).

La distancia al punto de vertido influyó de forma significativa los patrones en la estructura de la comunidad meiofaunal (PERMANOVA; Distancia, $P=0.0008$; tabla 3), aunque la magnitud de estas diferencias no fue consistente entre años (Distancia $\times$ Año, $P=0.0338$; tabla 3 ). Los dos primeros ejes del db-RDA explicaron aproximadamente el $45 \%$ de la variación total en la estructura de la comunidad meiofaunal (fig. 3). El primer eje estuvo positivamente correlacionado con el porcentaje de finos y negativamente correlacionado con la salinidad de la columna de agua; este eje acumuló un $28.5 \%$ de la variación total en la estructura de la comunidad meiofaunal. El segundo eje estuvo correlacionado negativamente con el porcentaje de arenas finas y el $\mathrm{pH}$ de la columna de agua, y acumuló un $15.8 \%$ de la variabilidad total de la estructura de la comunidad meiofaunal (fig. 3). Las cuatro variables ambientales anteriores (salinidad de la columna de agua, porcentajes de finos, $\mathrm{pH}$ de la columna de agua $\mathrm{y}$ porcentaje de arenas finas) fueron seleccionadas como las variables que más contribuyeron a explicar la variabilidad en

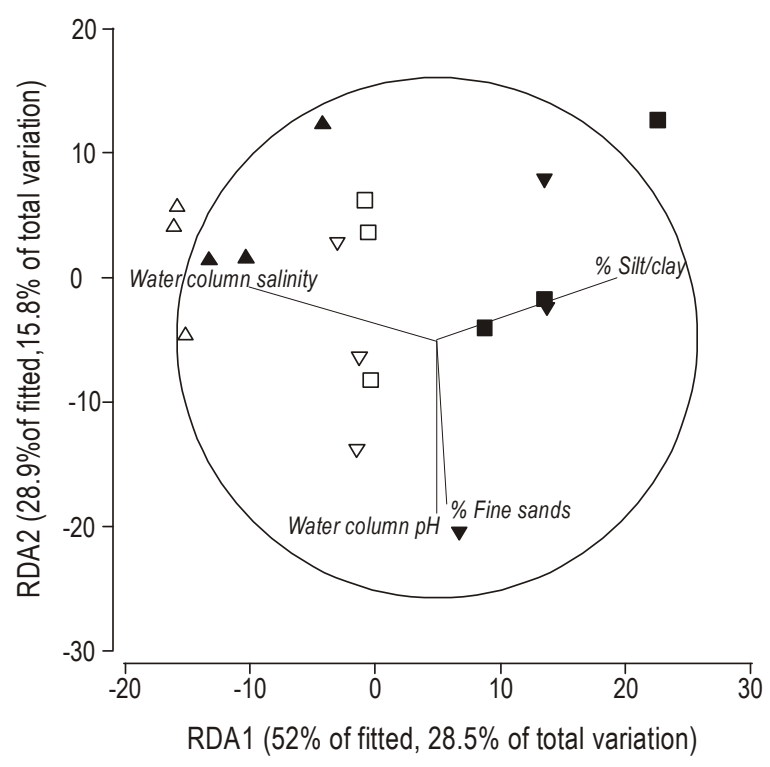

Figure 3. Distance-based redundancy analysis biplot of first and second axes relating those environmental variables that better explain the meiofaunal assemblage structure with varying proximity to the outfall (see table 1). Centroids for each distance and survey are plotted. $\boldsymbol{\Delta}: 0 \mathrm{~m}, \mathbf{m}: 15 \mathrm{~m}, \boldsymbol{\nabla}: 30 \mathrm{~m}$. White: May 2008; black: January 2009.

Figura 3. Análisis de redundancia basado en la distancia en dos dimensiones que relaciona aquellas variables ambientales que mejor explican la estructura de la comunidad meiofaunal según su proximidad al punto de vertido (ver tabla 1). Se representan los centroides de cada distancia y cada campaña de muestreo. $\boldsymbol{\Delta}: 0 \mathrm{~m}$, n: 15 m, $\boldsymbol{\nabla}: 30$ m. Blanco: mayo de 2008; negro: enero de 2009. 
and negatively correlated to the water column salinity, which accumulated $28.5 \%$ of the total variation in meiofaunal assemblage structure. The second axis was negatively correlated to the percentage of fine sands and the water column $\mathrm{pH}$, and accumulated $15.8 \%$ of the total variation in meiofaunal assemblage structure (fig. 3). The former four environmental variables (water column salinity, percentage of silt/clay, water column $\mathrm{pH}$, and percentage of fine sands) were selected as those variables that most contributed to explain the variation in meiofaunal assemblage structure (sequential tests in the multivariate multiple regression, table 4). A strong co-linearity was observed among several environmental variables. The percentage of silt/clay was negatively correlated with variables that denote the accumulation of waste products in sediments (table 5), while the water column salinity was positively correlated to the water column chlorophyll $a$ (table 5). Meiofaunal collections at $0 \mathrm{~m}$ were found on the left-hand side of the $\mathrm{db}-\mathrm{RDA}$, because they were (directly) positively correlated to the water column salinity (fig. 3), but also (indirectly) positively correlated to waste products in the sediment, as a result of the significant negative correlations between the percentage of silt/clay and the content of nitrogen and phosphorus, and percentage of organic matter (table 5). We also detected significant correlations among the different sediment types: coarse-sized fractions were negatively correlated to fine-sized fractions (table 5). It is worth noting, moreover, that different granulometric compositions with varying proximity to the discharge point were observed from May 2008 to January 2009 (table 1$)$. Gravels $(18.91 \pm 18.07 \%)$ and very coarse sands $(19.67 \pm 7.47 \%)$ were the most abundant granulometric fractions at $0 \mathrm{~m}$, whereas very fine sands dominated at $15 \mathrm{~m}$ $(57.32 \pm 8.10 \%)$ and $30 \mathrm{~m}(57.28 \pm 4.67 \%)$ from the disposal point in May 2008. In January 2009, however, fine sands and very fine sands were common at all distances (fine sands: $27.83 \pm 1.70 \%$ at $0 \mathrm{~m}, 31.85 \pm 7.64 \%$ at $15 \mathrm{~m}$, and $16.43 \pm$ $3.85 \%$ at $30 \mathrm{~m}$; very fine sands: $38.61 \pm 6.67 \%$ at $0 \mathrm{~m}, 31.80$ $\pm 2.49 \%$ at $15 \mathrm{~m}$, and $17.84 \pm 2.98 \%$ at $30 \mathrm{~m}$ ).

\section{DISCUSSION}

In this study, we observed a significant increase in meiofaunal abundances immediately adjacent (i.e., at $0 \mathrm{~m}$ ) to the combined sewage and brine outfall. Meiofaunal abundances decreased at a distance of 15 and $30 \mathrm{~m}$, reaching those values typically recorded for subtidal sandy seabeds of the Canary Archipelago (Riera 2004). Variations in meiofaunal abundances with varying proximity to the discharge point were concurrently accompanied by a change in meiofaunal assemblage structure.

The observational nature of our data does not allow an unequivocal evaluation of the separate and combined effects of each source of pollution on the meiofaunal assemblages. Results from this study, however, are in contrast with those recorded by Riera et al. (2011b) for a brine discharge point, la estructura de la comunidad meiofaunal (prueba secuencial en la regresión múltiple multivariante, tabla 4). Se observó una fuerte colinealidad entre varias variables ambientales. El porcentaje de finos estuvo correlacionado negativamente con variables que denotaron la acumulación de productos de desecho en sedimentos (tabla 5), mientras que la salinidad de la columna de agua estuvo correlacionada positivamente con la clorofila- $a$ (tabla 5). Las muestras meiofaunales a $0 \mathrm{~m}$ se dispusieron en la mitad izquierda del db-RDA porque estuvieron correlacionadas positivamente con la salinidad en la columna de agua (fig. 3), pero también indirectamente con la concentración de productos de desecho en el sedimento, como resultado de las correlaciones negativas significativas entre el porcentaje de finos y el contenido de nitrógeno, el contenido de fósforo y el porcentaje de materia orgánica (tabla 5). Además, cabe destacar, que la composición de las diferentes fracciones granulométricas varió entre las campañas según su proximidad al punto de vertido (tabla 1). En mayo de 2008 , las gravas $(18.91 \pm 18.07 \%)$ y las arenas muy gruesas $(19.67 \pm 7.47 \%)$ fueron la fracción granulométrica más abundante a $0 \mathrm{~m}$, mientras que las arenas muy finas dominaron a $15 \mathrm{~m}(57.32 \pm 8.10 \%)$ y $30 \mathrm{~m}(57.28 \pm 4.67 \%)$ de distancia del punto de descarga. Sin embargo, en enero de 2009 fueron abundantes las arenas finas y arenas muy finas en todas las distancias (arenas finas: $27.83 \pm 1.70 \%$ a $0 \mathrm{~m}$, $31.85 \pm 7.64 \%$ a $15 \mathrm{~m}, 16.43 \pm 3.85 \%$ a $30 \mathrm{~m}$; arenas muy finas: $38.61 \pm 6.67 \%$ a $0 \mathrm{~m} ; 31.80 \pm 2.49 \%$ a $15 \mathrm{~m} ; 17.84 \pm$ $2.98 \% 30 \mathrm{~m})$.

\section{DISCUSIÓN}

En este estudio se observó un aumento significativo de las abundancias meiofaunales inmediatamente adyacentes a $0 \mathrm{~m}$; es decir, el punto de descarga de salmuera y aguas domésticas residuales. Las abundancias meiofaunales disminuyeron a 15 y $30 \mathrm{~m}$ de distancia, alcanzando los valores típicos que se han registrado para fondos arenosos submareales del archipiélago Canario (Riera 2004). Estas variaciones en las abundancias meiofaunales según la proximidad al vertido estuvieron acompañadas de cambios en la estructura de la comunidad meiofaunal.

El carácter observacional de nuestros resultados no permite diferenciar entre los efectos de cada una de las fuentes de contaminación sobre las poblaciones meiofaunales. Sin embargo, los resultados de este estudio contrastan con los obtenidos por Riera et al. (2011b) para un punto de vertido exclusivo de salmuera, localizado a pocos kilómetros de distancia del punto de vertido combinado (salmuera y aguas residuales) que se muestreó en el presente estudio, utilizando una estrategia similar de muestreo. Riera et al. (2011b) observaron una disminución significativa en las abundancias meiofaunales inmediatamente adyacente al punto de vertido de salmuera, incluyendo bajas abundancias de nematodos. Este patrón se asoció al gradiente de salinidad observado en el punto de vertido, que varió entre $48(0 \mathrm{~m})$ y $36(30 \mathrm{~m})$. 
Table 4. Results of multivariate multiple regression testing the relationship between the measured set of environmental variables (see table 1) and the meiofaunal assemblage structure. To retain variables with explanatory power, the AIC procedure was chosen as model selection criterion (sequential tests, Legendre and Anderson 1999). $P$ values in bold denote significant values $(P<0.01)$. All data were fourth-root transformed.

Tabla 4. Resultados de regresión múltiple multivariante sobre las relaciones entre el conjunto de variables ambientales medidas (ver tabla 1) y la estructura de la comunidad meiofaunal. Para retener las variables con poder explicatorio, el procedimiento AIC fue elegido como modelo de selección de criterios (prueba secuencial, Legendre y Anderson 1999). Los valores de $P$ en negritas indican significación $(P<0.01)$. Todos los datos fueron transformados a la raíz a la cuarta.

\begin{tabular}{lccccc}
\hline Variable & AIC & SS (trace) & Pseudo- $F$ & $P$ & Proportion of explained variation \\
\hline Water column salinity & 109.240 & 1680.800 & 4.318 & $\mathbf{0 . 0 0 0 6}$ & 21.252 \\
Silt/clay (\%) & 106.850 & 1346.200 & 4.136 & $\mathbf{0 . 0 0 3 0}$ & 17.021 \\
Water column pH & 105.290 & 639.440 & 2.320 & 0.0650 & 0.547 \\
Fine sands (\%) & 106.240 & 659.300 & 2.186 & 0.0780 & 0.466 \\
\hline
\end{tabular}

located a few kilometers away from the combined sewage + brine discharge point of this study, using a similar sampling strategy. Riera et al. (2011b) observed a significant decrease in meiofaunal abundances immediately adjacent to the brine discharge point, including low abundances of nematodes, and associated this pattern with a salinity gradient ranging from 45 at $0 \mathrm{~m}$ to 36 at $30 \mathrm{~m}$ from the outfall. The salinity gradient observed in the present study was similar to the former study, ranging from 48 at $0 \mathrm{~m}$ to 36 at $30 \mathrm{~m}$ from the discharge point. Meiofaunal responses, however, were notably different: a significant increase in overall meiofaunal abundances, in particular nematodes, was observed immediately adjacent to the combined sewage + brine disposal point. As a result, it is plausible that the release of sewage overpowered the potential effects of brine on the meiofauna.

Sewage release may cause accumulations of particulate and dissolved organic carbon on the sediment (Perkins 1979), what can become an important food source for benthic organisms living in soft bottoms, such as nematodes (Gee and Warwick 1985), as long as levels of particulate and dissolved organic carbon do not surpass a threshold (Schratzberger and Warwick 1998). The larger content of organic matter at $0 \mathrm{~m}$ than at 15 and $30 \mathrm{~m}$ from the discharge point helps to explain the large abundance of nematodes at $0 \mathrm{~m}$. This was particularly relevant in May 2008, when coarse sediment fractions dominated at $0 \mathrm{~m}$ (coarse and very coarse sands, table 1 ) and nematode abundance decreased. Coarse sediments often harbor lower abundances of nematodes than seabeds dominated by finer sediments (Coull 1985); nematodes are endo- and epibenthic species mostly found in the first centimeters of fine-grained sediments (Platt 1989). Our results, therefore, are in accordance with previous studies where the abundance of nematodes increased notably due to their ability to exploit organic matter from domestic sewage discharges on sandy seabeds (Vidakovic 1983; Sandulli and De Nicola-Giudici $1990,1991)$, regardless of the seabed sedimentary composition. When sewage is discharged over other substrates, however, nematodes can respond differently. For example, Fraschetti et al. (2006) observed a decrease in nematode
Sin embargo, en el presente estudio las respuestas meiofaunales fueron totalmente diferentes; se observó un incremento significativo de las abundancias meiofaunales totales, en particular de nematodos, inmediatamente adyacente al punto combinado de vertido (salmuera y aguas residuales). Como resultado, es posible que el vertido de aguas residuales es más influyente que los efectos potenciales de la salmuera sobre la meiofauna.

El vertido de aguas residuales provoca acumulaciones de carbono orgánico disuelto y particulado en el fondo marino (Perkins 1979), y puede convertirse en una fuente importante de alimento para organismos bentónicos que viven en el sedimento, como los nematodos (Gee y Warwick 1985), siempre que no se supere un valor umbral para el carbono disuelto y particulado (Schartzberger y Warwick 1998). El contenido más elevado de materia orgánica a $0 \mathrm{~m}$, en comparación con los puntos a 15 y $30 \mathrm{~m}$ de distancia del punto de vertido, contribuye a explicar la mayor abundancia de nematodos. Este hecho fue particularmente relevante en mayo de 2008, cuando las fracciones gruesas de sedimento dominaron a $0 \mathrm{~m}$ (arenas gruesas y muy gruesas, tabla 1) y la abundancia de nematodos disminuyó. Los fondos con sedimentos gruesos suelen caracterizarse por abundancias más bajas de nematodos en comparación con fondos dominados por sedimentos más finos (Coull 1985); los nematodos son organismos endo- y epibentónicos, y se encuentran en los primeros centímetros de sedimentos de grano fino (Platt 1989). Por tanto, los resultados del presente estudio se encuentran en consonancia con los de estudios previos, donde se ha registrado que los nematodos incrementaron notablemente sus abundancias debido a su habilidad para asimilar la materia orgánica procedente de vertidos de aguas residuales en fondos arenosos (Vidakovic 1983, Sandulli y De Nicola-Giudici 1990, 1991), independientemente de su composición sedimentaria. Cuando se descargan las aguas residuales sobre otros sustratos, los nematodos pueden responder de forma diferente. Fraschetti et al. (2006), por ejemplo, observaron una disminución de las abundancias de nematodos en fondos adyacentes a un punto de vertido de aguas residuales. En 


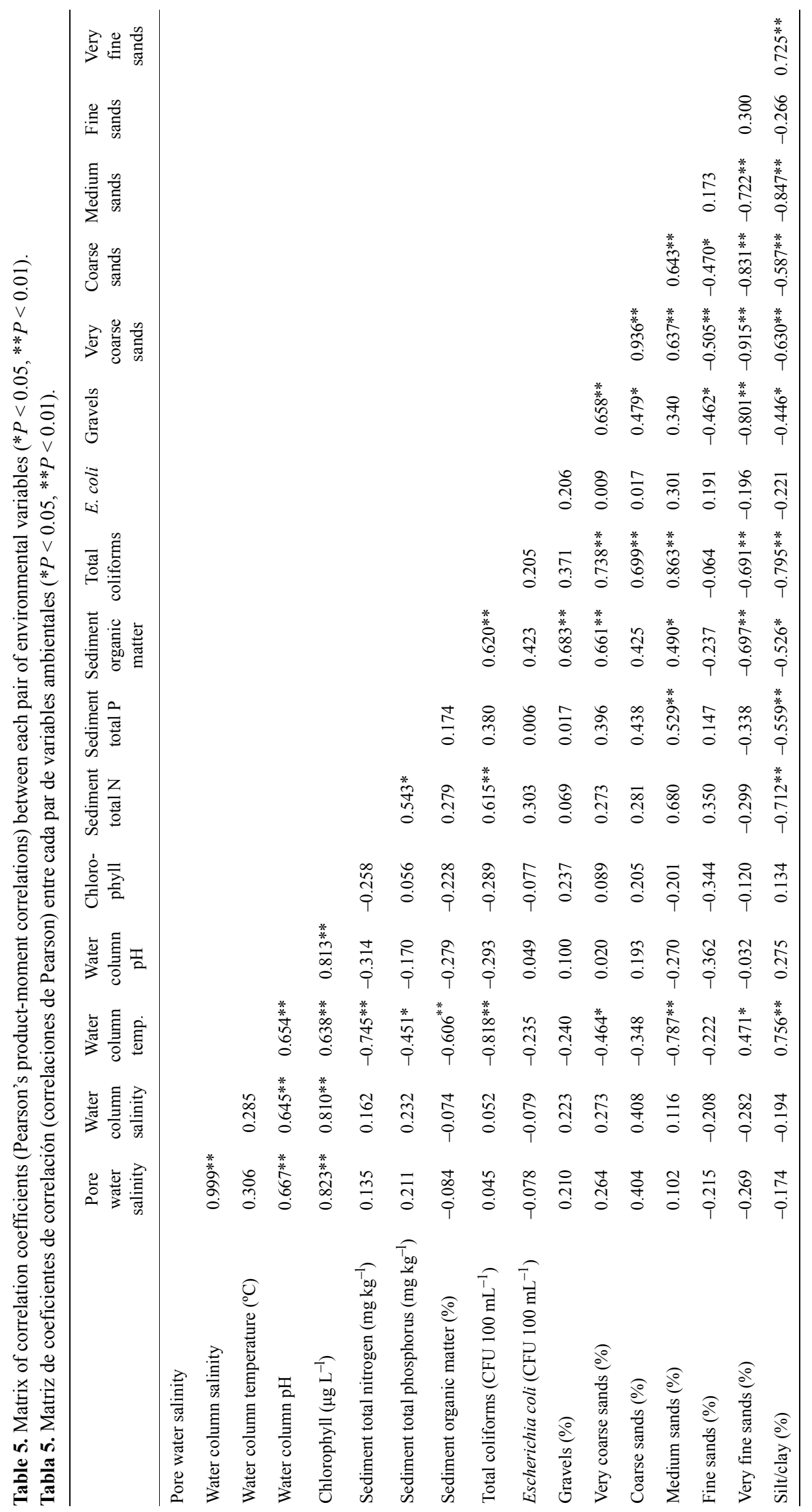


abundances adjacent to a domestic sewage discharge. In contrast to our study, where nematodes inhabited a sandy seabed, in that study nematodes inhabited a rocky substrate, where algal complexity most likely explains the changes in meiofaunal abundances (Terlizzi et al. 2005).

Benthic macroinvertebrate assemblages have been extensively used as bio-indicators of environmental disturbances, including both man-induced and natural perturbations (Borja et al. 2009). Meiofauna offer many advantages as indicators for ecological monitoring studies because of their small size, ubiquitous distribution, high diversity and abundances, in situ direct benthic development without a planktonic larval stage, and fast turnover rates (Schratzberger et al. 2000). In this study, we have demonstrated that meiofauna can be a suitable tool to monitor environmental impacts derived from combined sewage + brine outfalls on subtidal, sandy seabed assemblages. However, differences in the sediment (granulometric) composition may also notably influence patterns of meiofaunal assemblage structure, and so require careful consideration to avoid possible confounding interpretations for future monitoring assessment studies.

\section{ACKNOWLEDGEMENTS}

This study was supported by the Spanish Ministry for the Environment and Rural and Marine Affairs (project 056/ SGTB/2007/2.4). We would like to thank our colleagues J Costa and O Pérez (CIMA SL) for their constructive comments on an earlier draft of the manuscript and map preparation, as well as José Luis Sánchez-Lizaso (University of Alicante) for his insightful comments and suggestions throughout the study.

\section{REFERENCES}

Anderson MJ. 2001. A new method for non-parametric multivariate analysis of variance. Austral Ecol. 26: 32-46.

Austen MC, McEvoy AJ, Warwick RM. 1994. The specificity of meiobenthic community responses to different pollutants: Results from microcosm experiments. Mar. Pollut. Bull. 28: 557-563.

Azzurro E, Matiddi M, Fanelli E, Guidetti P, La Mesa G, Scarpato A, Axiak V. 2010. Sewage pollution impact on Mediterranean rocky-reef fish assemblages. Mar. Environ. Res. 69: 390-397. http://dx.doi.org/10.1016/j.marenvres.2010.01.006

Balestri E, Benedetti-Cecchi L, Lardicci C. 2004. Variability in patterns of growth and morphology of Posidonia oceanica exposed to urban and industrial wastes: Contrasts with two reference locations. J. Exp. Mar. Biol. Ecol. 308: 1-21.

Borja A, Muxica I, Rodríguez JG. 2009. Paradigmatic responses of marine benthic communities to different anthropogenic pressures, using M-AMBI, within the European Water Framework Directive. Mar. Ecol. 30, 214-227. http://dx.doi.org/10.1111/j.1439-0485.2008.00272.x

Bradstreet RB. 1965. The Kjeldahl Method for Organic Nitrogen. Academic Press, New York, 239 pp.

Burridge TR, Portelli T, Ashton P. 1996. Effects of sewage effluents on germination of three marine brown algal macrophytes. Mar. Freshwat. Res. 47: 1009-1014. contraste con nuestro estudio, donde se observó que los nematodos habitaron en fondos arenosos, estos autores observaron que los nematodos habitaron sobre un fondo rocoso; es probable que la complejidad algal explique los cambios en las abundancias meiofaunales (Terlizzi et al. 2005).

Las comunidades de invertebrados bentónicos se han utilizado de forma sistemática como bioindicadores de perturbaciones ambientales, incluyendo aquellas de origen antropogénico y de origen natural (Borja et al. 2009). La meiofauna ofrece una serie de ventajas como indicador para estudios de monitoreo ambiental debido a su pequeña talla, distribución ubicua, diversidad y abundancia elevadas, desarrollo bentónico directo sin fase larvaria pelágica y potencial reproductor alto (Schratzberger et al. 2000). En este estudio, se demostró que la meiofauna puede ser utilizada como una herramienta adecuada para los estudios de monitoreo ambiental en los vertidos combinados de salmuera y aguas residuales domésticas sobre comunidades de fondos arenosos submareales. Sin embargo, las diferencias en la composición granulométrica del sedimento pueden influir notablemente en los patrones de la estructura de la comunidad meiofaunal y, por tanto, requieren una evaluación cuidadosa para evitar posibles confusiones en la interpretación de los resultados de estudios de monitoreo ambiental.

\section{Agradecimientos}

Este estudio fue financiado por el Ministerio de Medio Ambiente y Medio Rural y Marino (España, proyecto 056/ SGTB/2007/2.4). Los autores agradecen a Janina Costa y Óscar Pérez (CIMA SL) por sus comentarios constructivos en una versión preliminar de este trabajo y a José Luis Sánchez-Lizaso (Universidad de Alicante) por sus sugerencias e ideas durante la realización de este estudio.

Traducido al español por los autores.

Butler R, Roddie BD, Mainstone CP, Wang TC, Tucker JW. 1990. The effects of sewage sludge on two life-history stages of Mytilus edulis. Chem. Ecol. 4: 211-219.

Chapman MG, Underwood AJ, Skilleter GA. 2005. Variability at different spatial scales between a subtidal assemblage exposed to the discharge of sewage and two control assemblages. J. Exp. Mar. Biol. Ecol. 189: 103-122.

Cotano U, Villate F. 2006. Anthropogenic influence on the organic fraction of sediments in two contrasting estuaries: A biochemical approach. Mar. Pollut. Bull. 52: 404-414.

Coull BC. 1985. Long-term variability of estuarine meiobenthos: A 11 year study. Mar. Ecol. Prog. Ser. 24: 205-218.

Danovaro R, Fabiano M, Vincx M. 1995. Meiofauna response to the Agip Abruzzo oil spill in subtidal sediments of the Ligurian Sea. Mar. Pollut. Bull. 30: 133-145.

Fraschetti S, Gambi C, Giangrande A, Musco L, Terlizzi A, Danovaro R. 2006. Structural and functional response of meiofaunal rocky assemblages to sewage pollution. Mar. Pollut. Bull. 52: 540-548. 
Gacia E, Invers O, Manzaner M, Ballesteros E, Romero J. 2007. Impact of the brine from a desalination plant on a shallow seagrass (Posidonia oceanica) meadow. Estuar. Coast. Shelf Sci. 72: 579-590.

Gee JM, Warwick RM. 1985. Effects of organic enrichment on meiofaunal abundance and community structure in sublittoral soft sediments. J. Exp. Mar. Biol. Ecol. 91: 247-262.

Gray JS. 1981. The Ecology of Marine Sediments. Cambridge University Press, Cambridge, $85 \mathrm{pp}$.

Grego M, De Troch M, Forte J, Malej A. 2009. Main meiofauna taxa as an indicator for assessing the spatial and seasonal impact of fish farming. Mar. Pollut. Bull. 58:1178-1186.

Guidetti P, Terlizzi A, Fraschetti S, Boero F. 2003. Changes in Mediterranean rocky-reef fish assemblages exposed to sewage pollution. Mar. Ecol. Prog. Ser. 253: 269-278.

Higgins RP, Thiel H. 1988. Introduction to the study of meiofauna. Smithsonian Institution Press, Washington, $488 \mathrm{pp}$.

Houlihan DF, Costello MJ, Secombes CJ, Stagg R, Brechin J. 1994. Effects of sewage sludge exposure on growth, feeding and protein-synthesis of dab (Limanda limanda (L)). Mar. Environ. Res. 37: 331-353.

Korajkic A, Brownell MJ, Harwood VJ. 2010. Investigation of human sewage pollution and pathogen analysis at Florida Gulf coast beaches. J. Appl. Microbiol. 110: 174-183.

Legendre P, Anderson MJ. 1999. Distance-based redundancy analysis: Testing multispecies responses in multifactorial ecological experiments. Ecol. Monogr. 69: 1-24.

Mirto S, La Rosa T, Gambi C, Danovaro R, Mazzola A. 2000. Microbial and meiofaunal response to intensive mussel-farm biodeposition in coastal sediments of the western Mediterranean. Mar. Pollut. Bull. 40: 244-252. http://dx.doi.org/10.1016/S0025-326X(99)00209-X

Murphy J, Riley JP. 1962. A modified single solution method for the determination of phosphate in natural waters. Anal. Chem. Acta 27: $31-36$.

Oviatt CA, Quinn JG, Maughan JT, Ellis JT, Sullivan BK, Gearing JN, Gearing PJ, Hunt CD, Sampou PA, Latimer JS. 1987. Fate and effects of sewage sludge in the coastal marine environment: A mesocosm experiment. Mar. Ecol. Prog. Ser. 41: 187-203.

Pastorok RA, Bilyard GR. 1985. Effects of sewage pollution on coral-reef communities. Mar. Ecol. Prog. Ser. 21: 175-189.

Pearson TH, Rosenberg R. 1978. Macrobenthic succession in relation to organic enrichment and pollution of the marine environment. Oceanogr. Mar. Biol. Annu. Rev. 16: 229-311.

Perkins EJ. 1979. The effects of marine discharges on the ecology of coastal waters. In: James A, Evison L (eds.), Biological Indicators of water quality. Wiley, New York, pp. 41-67.

Platt HM. 1989. The significance of free-living marine nematodes in the ecosystems. Zool. J. Linn. Soc. 96: 261-279.

Reopanichkul P, Schlacher TA, Carter RW, Worachanant S. 2009. Sewage impacts coral reefs at multiple levels of ecological organization. Mar. Pollut. Bull. 58: 1356-1362.

Riera R. 2004. Biodiversidad meiofaunal de las playas de Los Abrigos del Porís y de Los Cristianos en la isla de Tenerife. Estructura y dinámica de sus comunidades. $\mathrm{PhD}$ thesis, University of La Laguna, 486 pp.
Riera R, Monterroso O, Rodríguez M, Ramos E, Sacramento A. 2011a. Six-year study of meiofauna dynamics. Aquat. Ecol. 45: 221-229.

Riera R, Tuya F, Sacramento A, Ramos E, Rodríguez M, Monterroso O. 2011b. A community level assessment to evaluate the effects of brine disposal over subtidal meiofauna. Estuar. Coast. Shelf Sci. 93: 359-365.

Roberts DA, Johnston EL, Knott NA. 2010. Impacts of desalination plant discharges on the marine environment: A critical review of published studies. Water Res. 44: 5117-5128.

Sadhwani J, Veza J, Santana C. 2005. Case studies on environmental impact of seawater desalination. Desalination 185: 1-8.

Sandulli R, De Nicola-Giudici M. 1990. Pollution effects on the structure of meiofaunal communities in the Bay of Naples. Mar. Pollut. Bull. 21: 144-153.

Sandulli R, De Nicola-Giudici M. 1991. Responses of meiobenthic communities along a gradient of sewage pollution. Mar. Poll. Bull. 22: 463-467. http://dx.doi.org/10.1016/0025-326X(91)90217-G

Schratzberger M, Warwick RM. 1998. Effects of the intensity and frequency of organic enrichment on two estuarine nematode communities. Mar. Ecol. Prog. Ser. 164: 83-94.

Schratzberger M, Gee JM, Rees HL, Boyd SE, Wall CM. 2000. The structure and taxonomic composition of sublittoral meiofauna assemblages as an indicator of the status of marine environments J. Mar. Biol. Assoc. UK 80(6): 969-980.

Smith J, Shackley SE. 2006. Effect of the closure of a major sewage outfall on sublittoral, soft sediment benthic communities. Mar. Pollut. Bull. 52: 445-458.

Somerfield P, Warwick RM. 1996. Meiofauna in marine pollution programmes. A laboratory manual. Ministry of Agriculture, Fisheries and Food, Directorate of Fisheries Research, Lowestoft, UK, $71 \mathrm{pp}$.

Terlizzi A, Fraschetti S, Guidetti P, Boero F. 2002. The effects of sewage discharge on shallow hard substrate sessile assemblages. Mar. Pollut. Bull. 44: 544-550.

Terlizzi A, Benedetti-Cecchi L, Bevilacqua S, Fraschetti S, Guidetti P, Anderson MJ. 2005. Multivariate and univariate asymmetrical analyses in environmental impact assessment: A case study of Mediterranean subtidal sessile assemblages. Mar. Ecol. Prog. Ser. 289: 27-42.

Thornberg CS, DiMilla P, Nixon SW, McKinney RA. 2008. Natural and anthropogenic nitrogen uptake by bloom-forming macroalgae. Mar. Pollut. Bull. 56: 261-269. http://dx.doi.org/10.1016/j.marpolbul.2007.10.031

Underwood AJ. 1991. Beyond BACI: Experimental designs for detecting human impacts on temporal variations in natural populations. Aust. J. Mar. Freshw. Res. 42: 569-587. http://dx.doi.org/10.1071/MF9910569

Vidakovic J. 1983. The influence of raw domestic sewage on density and distribution of meiofauna. Mar. Pollut. Bull. 14: 84-88.

Walkley A, Black JA. 1934. An examination of the Degtjareff method for determining soil organic matter and a proposed modification of the chromic titration method. Soil Sci. 37: 29-38.

http://dx.doi.org/10.1097/00010694-193401000-00003

Received May 2012,

received in revised form October 2012, accepted November 2012. 